\title{
Lactobacillus reuteri reduces episodes of diarrhoea in healthy children
}

A new study published in Pediatrics demonstrates that daily administration of the probiotic strain Lactobacillus reuteri DSM 17938 to healthy children reduces episodes of acute diarrhoea. In addition, the treatment conferred a cost-saving benefit to the families and health-care system.

Healthy children attending day care are at increased risk of contracting diarrhoea compared with those cared for at home. A growing body of research has

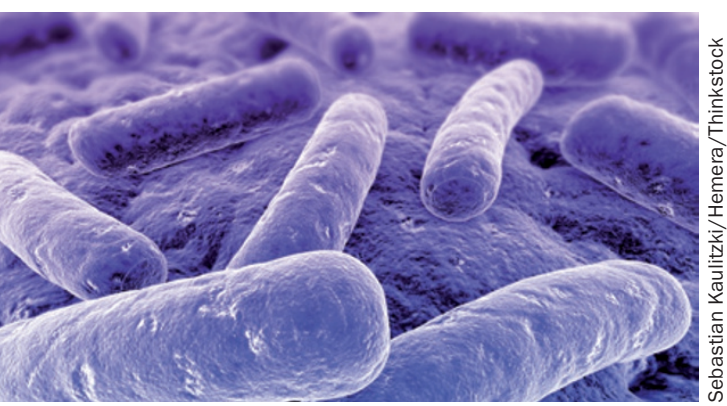

demonstrated benefits of administering probiotics to this population. This progress led Pedro Gutierrez-Castrellon and colleagues to investigate the use of L. reuteri as a prophylactic treatment in preschool children in Mexico.

The investigators enrolled 336 children aged 6-36 months from different day-care centres in Mexico City and randomly assigned them to receive either a suspension of L. reuteri DSM 17938 $\left(1 \times 10^{8}\right.$ colony-forming units $)$ or placebo (no probiotic strain included) each day. Children were treated for 3 months, with additional data collected 3 months after treatment concluded.

The investigators assessed a number of health and social outcomes, such as the number of medical visits and absenteeism, but the primary focus was on documenting the frequency and duration of episodes of diarrhoea and respiratory tract infection.
A substantial reduction in the average incidence and duration of diarrhoea and respiratory tract infection was seen in the treatment group at both 3 and 6 months. Investigators also performed a cost-effectiveness analysis demonstrating that treament provides a direct economic benefit. No adverse events were reported with the probiotic strain.

Currently, probiotics are not recommended as an adjuvant treatment for acute community-aquired diarrhoea; however, research into their use is increasing. The health and socioeconomic benefits found in this study could inform future reccomendations to shape management of childhood diarrhoea in low-income and middle-income countries. Gillian Patman

Original article Gutierrez-Castrellon, P. et al. Diarrhea in preschool children and Lactobacillus reuteri: a randomized control trial. Pediatrics doi:10.1542/peds.2013-0652 